\title{
Integração espacial e transmissão de preços das cestas básicas entre as capitais da região sul do Brasil
}

Claudeci da Silva*

Walberti Saith ${ }^{* *}$

Resumo: Fundamentado na teoria de integração de mercado e da Lei de Preço Único, este artigo analisa a integração espacial e transmissão de preços da cesta básica nas três capitais da região sul - Curitiba-PR, Florianópolis-SC e Porto Alegre-RS - no período de janeiro de 2000 a janeiro de 2011. Nas análises metodológicas foram utilizados testes de raiz unitária, teste de causalidade Granger, testes de cointegração de Johansen e, por fim, foram estimados modelos de Correção de Erro Vetorial (VEC). Com base nos modelos VEC pode-se concluir que existe alto grau de integração e que as elasticidades dos preços de longo prazo são altas, embora existam desvios de curto prazo.

Palavras-chave: Cesta básica; Integração de mercados; Cointegração; Lei de Preço Único.

Classificação JEL: C32; D41; R12.

\footnotetext{
“Mestranda em Teorias Econômicas pela Universidade Estadual de Maringá (UEM). Endereço eletrônico: chardeci@ bol.com.br.

** Mestrando em Teorias Econômicas pela Universidade Estadual de Maringá (UEM). Endereço eletrônico: saith@bol. com.br.
} 


\section{Introdução}

Os estudos sobre integração espacial e transmissão de preços abordam um importante aspecto da economia: a distribuição espacial de bens. Dentro da discussão sobre integração de mercado, a cesta básica destaca-se por representar um conjunto de produtos necessários a sobrevivência do trabalhador e, também, por ser um item importante na composição de índices de inflação.

A pesquisa da Cesta Básica Nacional (Ração Essencial Mínima), realizada pelo Departamento Intersindical de Estatística e Estudos Socioeconômicos (DIEESE), em dezesseis capitais do Brasil, acompanha mensalmente a evolução de preços de treze produtos de alimentação. A metodologia utilizada pelo DIEESE para a Pesquisa da Cesta Básica Nacional foi estabelecida com base no Decreto Lei $\mathrm{n}^{\circ} 399$, que regulamenta o salário mínimo. No Brasil, o critério utilizado para orientar o valor do salário mínimo deve ser tal para que este garanta a compra da cesta básica.

A região sul é importante para a economia brasileira e abriga uma parcela considerável da população. Tendo em vista a importância da cesta básica e o papel da região sul na economia, o presente trabalho tem como objetivo entender as relações causais de transmissão de preços e cointegração espacial da cesta básica entre as capitais desta região, ou seja, Curitiba-PR, Florianópolis-SC e Porto Alegre-RS.

\section{Referencial teórico}

A discussão acerca de mercados integrados é proposta pela Lei de Preço Único (LPU); segundo esta, os bens homogêneos disponíveis em diversos mercados, de regiões distintas, seguem o mecanismo de arbitragem, o qual garante que os preços entre esses mercados - quando expresso em mesma moeda, em se tratamento do mercado internacional - não podem diferir entre si acima do valor de seus custos de transação (Costa, Ferreira Filho, 2000; Rosado, Maciel, Lima, 2008).

No curto prazo há possibilidades de ocorrer desvios ou desajustes entre os valores, até mesmo acima do valor dos custos de transação, mas no longo prazo estes tendem a convergir para os verdadeiros valores mantendo a relação limite descrita anteriormente (Costa, Ferreira Filho, 2000). Matematicamente Revista Economia \& Tecnologia (RET) Vol. 8(1), p. 87-96, Jan/Mar 2012 
esta relação pode ser expressa como:

$$
P_{i t}-P_{j t} \leq r_{i j}
$$

onde a variável $P_{i t}$ representa o preço de um bem na região $i, P_{j t}$ na região $j$ e $r_{i j}$ representa o custo de transporte, para deslocar o bem de uma região a outra. $\mathrm{O}$ equilíbrio representado na Equação (1) é denominado de condição de arbitragem espacial.

A Lei do Preço Único (LPU) mostra a relação de equilíbrio de longo prazo entre preços estabelecidos em duas ou mais regiões distintas. A mudança do preço em uma região tende a conduzir mudanças nos preços de equilíbrio das demais regiões relacionadas a esta. Em se tratando de relações entre países, a LPU pode ser expressa como:

$$
P_{i t}=a+b P_{j t}+u_{t}
$$

As variáveis $P_{i t}$ e $P_{j t}$ representam o preço de uma commodity nos mercados do país $i$ e $j$. A variável $a$ representa uma constante e $u_{t}$ representa um fator de erro.

Valendo a LPU, no longo prazo, oscilações de preço em um mercado serão transmitidas proporcionalmente aos demais mercados, de modo que a elasticidade de transmissão de preço, representado por $b$ na Equação (2), tende a ser igual a um (Cunha, Quintela, Gomes, Rufino, 2007). Se o valor do coeficiente for estatisticamente igual a zero isto implica que variações de preço no país $j$ não conduzem a qualquer tipo de reação do preço no país $i$, neste caso pode-se dizer que a economia deste país é completamente fechada.

Um alto grau de integração não ocorre apenas na presença de comércio direto entre as regiões, a transmissão de preços pode ocorrer também nos mercados que se ligam indiretamente via ligações comerciais a um terceiro mercado em comum.

Vale ressaltar que na presença de altos custos de transportes ou se houver barreiras ao comércio de mercadorias a integração pode não ocorrer. (Pippenger, Phillips, 2007). 


\section{Metodologia}

As séries usadas neste trabalho são do DIEESE e compreendem o período de janeiro de 2000 a janeiro de 2011. Será analisado o preço da cesta básica nas três capitais da região sul: Curitiba-PR, Florianópolis-SC e Porto Alegre-RS. Em todas as séries foi aplicado o logaritmo natural para facilitar os cálculos econométricos.

Para analisarmos a integração espacial primeiramente precisamos determinar a ordem de integração das séries. Neste trabalho utilizaremos dois testes, Dickey-Fuller aumentado e KPSS, muito utilizados em estudos de séries temporais.

Em seguida realiza-se o teste de causalidade proposto por Granger em 1969, que parte da seguinte premissa: se uma variável X causa Y, logo variações em $\mathrm{X}$ deveriam anteceder variações em $\mathrm{Y}$.

A sentença "X causa Y" demanda duas condições: primeira, $\mathrm{X}$ deve ajudar a prever Y; e, segunda, Y não deve ajudar a prever X. A causalidade testada deste modo não tem o significado estrito da palavra, mas é uma ferramenta importante no entendimento do sentido da variação de preço.

Definido o sentido de causalidade entre as séries analisadas, o próximo passo é analisar a existência de cointegração entre estas. Para testar a existência de equilíbrio de longo prazo entre as variáveis adota-se a metodologia desenvolvida por Johansen, que propõe dois testes diferentes de relação de significância para a estimação por máxima verossimilhança da combinação canônica: o teste do traço, que assume como hipótese nula a existência de $r$ vetores de cointegração contra a hipótese alternativa de $r>r^{*}$ vetores, e o teste do máximo autovalor, que testa a existência de $r$ vetores de cointegração contra a alternativa de existência de $r+1$ vetores.

Uma regressão por Mínimos Quadrados Ordinários (MQO) de uma série temporal não estacionária contra outra série temporal não estacionária poderia produzir uma regressão espúria. Todavia, se os resíduos resultantes dessa regressão forem estacionários existe uma relação de longo prazo entre as séries, pois embora as séries sejam I(1), sua combinação linear é I(0). A combinação linear faz com que as tendências estocásticas das duas séries se anulem; como resultado, tem-se que as variáveis em análise são cointegradas. 
A relação da equação cointegrante mostra um equilíbrio de longo prazo entre as variáveis, podendo no curto prazo ocorrer desequilíbrios; por conseguinte, o termo de erro da Equação (3) por ser entendido como um "erro de equilíbrio". O mecanismo de correção de erros pode ser visto como um elo entre o curto e o longo prazo entre as variáveis. Se duas variáveis são cointegradas, sua relação pode ser expressa como um mecanismo de correção de erros do seguinte modo:

$$
\Delta P_{t}^{i}=\alpha_{1}+\alpha_{2} \Delta P_{t-1}^{i}+\alpha_{3} \Delta P_{t-1}^{j}+\alpha_{4} \mu_{t-1}+\varepsilon_{t}
$$

onde $\Delta$ é o operador de diferença; $\varepsilon_{t}$ é um termo de erro aleatório; e $\mu_{t-1}$ é o termo de erro defasado da regressão cointegrante. Propõe-se uma normalização aos vetores de cointegração, de forma a restringir as possibilidades que essas matrizes podem assumir (Bueno, 2008).

Os coeficientes $\alpha_{2}$ e $\alpha_{3}$ captam as relações de curto prazo entre as variáveis e $\alpha_{4}$ representa a relação de longo prazo. Se o último for diferente de zero o modelo está fora de equilíbrio e o vetor de correção de erros corrigirá o sistema no período seguinte, assim $\alpha_{4}$ decide o quão rapidamente o equilíbrio é restaurado. Caso o termo de erro de equilíbrio seja estatisticamente igual a zero, a variável dependente se ajusta a mudanças na variável independente no mesmo período, portanto não existe desvio da relação de longo prazo.

\section{Resultados}

A evolução do preço da cesta básica, entre o período que vai de janeiro de 2000 a janeiro de 2011, nas três capitais da região sul, foram plotados no Gráfico 1.

Nota-se que as séries não apresentam uma tendência linear bem definida ao longo do tempo; no entanto, elas se movimentam conjuntamente em uma mesma tendência, sugerindo que estas sejam cointegradas, estabelecendo uma relação de equilíbrio de longo prazo. Para verificar a existência de cointegração é necessário que as séries cumpram alguns requisitos conforme serão analisados a seguir. 
Gráfico 1 - Evolução nominal dos preços da cesta básica de 2000 a 2011

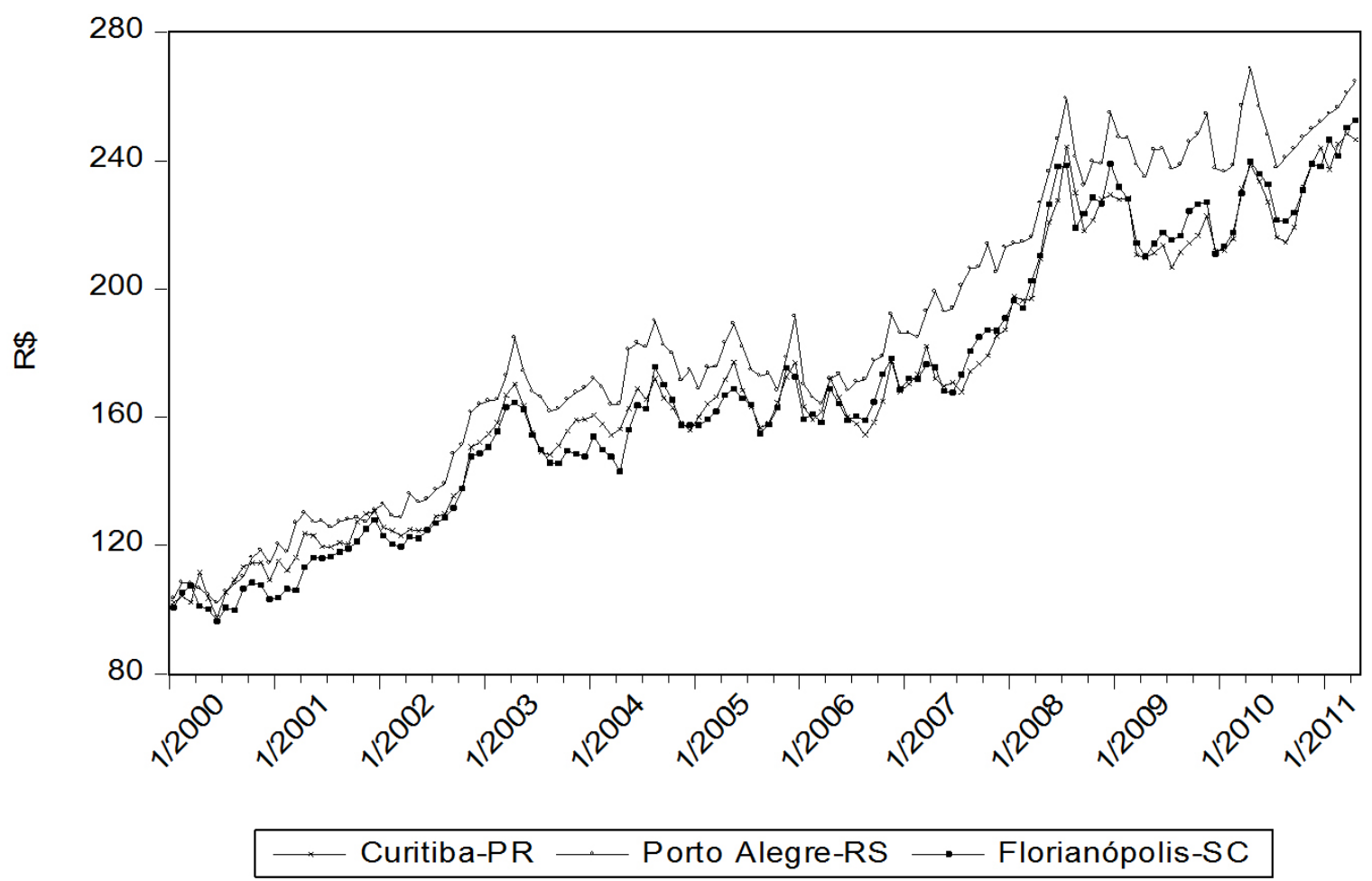

Fonte: DIEESE (elaboração própria).

Na Tabela 1 estão os resultados dos testes de raiz unitária. O número de defasagem dos modelos foram selecionados a partir do critério de informação; optou-se por utilizar o número de defasagens que minimizassem o Critério Akaike (AIK) e o Critério de Informação Hannan-Quinn (HQIC). Os testes, Dickey-Fuller e KPSS, mostram que ao nível de significância de 1\% as séries em questão são todas estacionárias em primeira diferença, sendo assim as mesmas pode ser ditas integradas de ordem um I(1) e é possível que estas séries sejam cointegradas.

Tabela 1 - Teste de raiz unitária

\begin{tabular}{|c|c|c|c|c|c|}
\hline \multirow{2}{*}{ Variável } & \multirow{2}{*}{ Defasagens } & \multicolumn{2}{|c|}{ Dickey-Fuller } & \multicolumn{2}{|r|}{ KPSS } \\
\hline & & Modelo & Estatística do teste & Modelo & Estatística do teste \\
\hline$\overline{\mathrm{PR}}$ & 4 & Tend.Const. & $-2,649$ & Constante & $2,590^{*}$ \\
\hline$\Delta \mathrm{PR}$ & 3 & Const & $-7,567^{*}$ & Constante & 0,045 \\
\hline $\mathrm{SC}$ & 4 & Tend.Const. & $-2,391$ & Constante & $2,630^{*}$ \\
\hline$\Delta \mathrm{SC}$ & 3 & Const & $-7,740^{*}$ & Constante & 0,037 \\
\hline RS & 1 & Tend.Const. & $-2,869$ & Constante & $6,300^{*}$ \\
\hline$\Delta \mathrm{Rs}_{\mathrm{s}}$ & 3 & Const. & $-7,730^{*}$ & Constante & 0,090 \\
\hline
\end{tabular}

Fonte: Elaboração própria.

Nota: ${ }^{*}$ Significativo a $1 \%$.

A direção em que se dá a causalidade ou a transmissão pode ser 
observada por meio do teste de Granger. Os resultados do teste estão compilados na Tabela 2.

No curto prazo o preço da cesta básica no Rio Grande do Sul é afetado pelo preço da cesta básica do Paraná e por Santa Catarina. O Rio Grande do Sul por sua vez causa o Paraná havendo, portanto, uma relação bicausal. Já Santa Catarina não é causada por nenhuma outra variável, o que pode ser uma evidência de que esta região seja a principal região formadora do preço. Recordando que o significado de causalidade nesse teste é o de precedência.

Tabela 2 - Teste de causalidade de Granger

\begin{tabular}{lc|c|c}
\hline \multicolumn{2}{l}{ Direção da causalidade } & Estatística $\mathbf{F}$ & Prob. $>$ F \\
\hline$\Delta \mathrm{PR}$ & $\Delta \mathrm{SC}$ & 10,066 & 0,0001 \\
$\Delta \mathrm{PR}$ & $\Delta \mathrm{RS}$ & 0,805 & 0,4495 \\
$\Delta \mathrm{SC}$ & $\Delta \mathrm{PR}$ & 2,842 & 0,0621 \\
$\Delta \mathrm{SC}$ & $\Delta \mathrm{RS}$ & 1,173 & 0,3129 \\
$\Delta \mathrm{RS}$ & $\Delta \mathrm{PR}$ & 1,344 & 0,2645 \\
$\Delta \mathrm{RS}$ & $\Delta \mathrm{SC}$ & 7,144 & 0,0012 \\
\hline \multicolumn{2}{l}{} \\
\hline \multicolumn{2}{l}{ Fonte: Elaboração própria. }
\end{tabular}

Fonte: Elaboração própria.

Uma vez verificada que as séries são integradas da mesma ordem e realizado o teste de causalidade, o passo seguinte é testar se estas séries são cointegradas através do procedimento de Johansen, que toma por base a especificação de um modelo VAR. A existência de um vetor de cointegração entre as séries permitirá a convergência no longo prazo. A prova da existência de vetores de cointegração entre as séries é efetuada mediante o teste do traço e de teste de máximo autovalor. Seus resultados estão expostos na Tabela 3.

Tabela 3 - Teste de cointegração de Johansen

\begin{tabular}{l|c|r|r|r}
\hline $\begin{array}{l}\mathbf{N}^{\mathbf{o}} \text { vetores de } \\
\text { cointegração }\end{array}$ & Traço & $\begin{array}{c}\text { Valor crítico } \\
\mathbf{( 5 \% )}\end{array}$ & $\begin{array}{c}\text { Max. } \\
\text { autovalor }\end{array}$ & $\begin{array}{c}\text { Valor crítico } \\
\mathbf{( 5 \% )}\end{array}$ \\
\hline $\mathrm{r}=0$ & 19.8949 & 15,41 & 18,0405 & 14,07 \\
$\mathrm{r} \leq 1$ & $1.8544^{*}$ & 3,76 & 1,8544 & 3,76 \\
\hline
\end{tabular}

Fonte: Elaboração própria.

Nota: "Estatisticamente significativo.

Com base nos testes, pode-se concluir que as séries apresentam pelo menos um vetor de cointegração que as une no longo prazo. Comprovada a existência de uma relação de longo prazo entre as variáveis, estimam-se as elasticidades de longo prazo por meio de MQO. As equações cointegrantes são apresentadas na Tabela 4 . 
Tabela 4 - Coeficientes da equação cointegrante

\begin{tabular}{l|c|r|r|r}
\hline & Constante & \multicolumn{1}{c|}{ PR } & \multicolumn{1}{c|}{ RS } & \multicolumn{1}{c}{ SC } \\
\hline PR & $-0,444780$ & 1 & 0,064807 & $-0,980697^{*}$ \\
RS & $-6,863161$ & $15,43045^{*}$ & 1 & $-15,13259^{*}$ \\
SC & 0,453535 & $-1,01968^{*}$ & $-0,066083$ & 1 \\
\hline
\end{tabular}

Fonte: Elaboração própria.

Nota: "Significativo a $5 \%$.

As três variáveis foram consideradas como sendo endógenas. No primeiro caso, Curitiba-PR é considerada como sendo endógena e somente o coeficiente referente à Florianópolis-SC é estatisticamente significativo. $\mathrm{Na}$ equação onde Florianópolis-SC é considerado como sendo endógeno, somente o coeficiente relativo à Curitiba-PR é estatisticamente significativo, evidenciando uma relação bicausal entre essas duas regiões.

Quando Porto Alegre-RS é considerada a variável explicativa, esta não é estatisticamente significativa em nenhum caso e, na ocasião em que é considerada como endógena, todas as variáveis explicativas foram significativas estatisticamente, portanto há evidências de que o preço da cesta básica em Porto Alegre-RS seja determinado pelo preço nas outras capitais da região sul. A análise dos desvios de curto prazo é realizada por meio do modelo VEC. Os coeficientes deste modelo estão compilados na Tabela 5.

\begin{tabular}{lrrrr}
\multicolumn{4}{c}{ Tabela 5 - Coeficientes do modelo VEC } \\
\hline & \multicolumn{1}{c}{$\Delta$ PR } & \multicolumn{1}{c}{$\Delta \mathbf{R S}$} & \multicolumn{1}{c}{$\Delta$ SC } \\
\hline$\Delta \mathrm{PR}_{t-1}$ & 0,084396 & 0,198279 & 0,173764 \\
$\Delta \mathrm{RS}_{t-1}$ & $-0,147766$ & $-0,436840^{*}$ & $-0,205919$ \\
$\Delta \mathrm{SC}_{t-1}$ & $0,295709^{*}$ & $0,418761^{*}$ & 0,178871 \\
Constante & 0,004884 & 0,005517 & 0,005583 \\
$\alpha_{4}(\mathrm{PR})$ & $-0,367721^{*}$ & $-0,023831^{*}$ & $0,360623^{*}$ \\
$\alpha_{4}(\mathrm{RS})$ & $-0,033358$ & $-0,002162$ & 0,032714 \\
$\alpha_{4}(\mathrm{SC})$ & 0,157731 & 0,010222 & $-0,154686$ \\
$\mathrm{R}^{2}$ & 0,157295 & 0,145559 & 0,067903 \\
Log likelihood $_{\text {Teste F }}$ & 267,9304 & 272,8954 & 270,2171 \\
Fonte: Elaboração própria. & 6,019606 & 5,493973 & 2,349404 \\
Nota: ${ }^{*}$ Significativo a $5 \%$. & &
\end{tabular}

Os resultados do modelo VEC multivariado indicam que o preço na cidade de Curitiba-PR é uma variável dependente principalmente do preço praticado em Florianópolis-SC. Já a cesta básica em Florianópolis-SC mostrouse independente, já que nenhum dos coeficientes de longo prazo na equação em que esta figura como variável dependente foram estatisticamente significativos. O preço da cesta básica em Porto Alegre-RS é afetado pelos preços de CuritibaRevista Economia \& Tecnologia (RET) 
PR e Florianópolis-SC, sendo que estes não são afetados pelo primeiro, indicando assim que existe transmissão de preço unidirecional.

\section{Considerações finais}

Os resultados mostraram que, em relação à hipótese levantada inicialmente neste trabalho, existem evidências empíricas que indicam a existência de integração espacial entre os preços da cesta básica nas capitais da região sul. As elasticidades de longo prazo nos casos em que Porto Alegre-RS figura como variável explicativa não foram estatisticamente significativas, ou seja, não existe transmissão desta para as demais regiões. Já na relação entre Curitiba-PR e Florianópolis-SC, os coeficientes das elasticidades de longo prazo revelaram que existe uma transmissão de preços bidirecional.

Em estudos futuros sobre integração espacial, além das dimensões aqui estudadas, outras regiões deveriam ser levadas em consideração, como é o caso da região sudeste, que devido à proximidade pode influenciar a região sul. Outro aspecto que poderia ser explorado são os custos de transações, que são de suma importância na compreensão do comércio regional.

\section{Referências}

Carvalho, Henrique Duarte; Scalco, Paulo Roberto; Lima, João Eustáquio de. (2009). "Integração espacial entre os preços das cestas básicas nas capitais da região sudeste do Brasil". Revista Economia 10(2): 373-399.

Costa, Silvia Maria A. Lima; Ferreira Filho, Joaquim Bento de Souza. (2000). "Liberação comercial no Brasil e integração nos mercados de commodities agrícolas: Os mercados do algodão, milho e arroz". Revista de Economia e Sociologia Rural 38(2): 41-70.

Cunha, Dênis Antônio; Quintela, Mirelle Cristina de Abreu; Gomes, Marília Maciel; Rufino, José Luíz dos Santos. (2007). "Integração dos preços ao produtor e preços da Bolsa de Nova Iorque para o café arábica brasileiro". Anais do $45^{\circ}$ Congresso da Sociedade Brasileira de Economia, Administração e Sociologia Rural.

Goodwin, Barry K.; Piggott, Nicholas E. (1999) "Spatial market integration in the presence of threshold effects". Research in Agricultural and Applied Economics Annual Meeting.

Pippenger, John; Phillips, Llad. (2007). "Strictly speaking, the law of on price works in commodity markets". University of California, Working paper n. 16-07.

Rosado, Patricia Lopes; Maciel, Marília Fernandes; Lima, João Eustáquio. (2008). 
Claudeci da Silva, Walberti Saith

"Análise da estrutura espacial do mercado brasileiro de suínos". Anais do $46^{\circ}$ Congresso da Sociedade Brasileira de Economia, Administração e Sociologia Rural. 